\title{
Preface
}

\section{The variation of marine environment and climate effect in Indo-Pacific Ocean}

\author{
ZUO Juncheng ${ }^{1,{ }^{*}}$, WANG Weiqiang ${ }^{2}$, WANG Guihua ${ }^{3}$, XIE Qiang ${ }^{4}$ \\ ${ }^{1}$ Marine Science and Technology College, Zhejiang Ocean University, Zhoushan 316022, China \\ ${ }^{2}$ State Key Laboratory of Tropical Oceanography, South China Sea Institute of Oceanology, Chinese Academy of Sciences, \\ Guangzhou 510301, China \\ ${ }^{3}$ Institute of Atmospheric Sciences, Fudan University, Shanghai 200438, China \\ ${ }^{4}$ Institute of Deep-sea Science and Engineering, Chinese Academy of Sciences, Sanya 572000, China
}

Received Sep. 24, 2020; accepted for publication Sep. 30, 2020

(C) Chinese Society for Oceanology and Limnology, Science Press and Springer-Verlag GmbH Germany, part of Springer Nature 2020

In the background of global change, the variability of the marine environment and the resulting climate effects in the Asia-Pacific region and the Indian Ocean have become increasingly prominent. The research on climate effects has great research significance and national needs. In order to track and discuss major scientific and technological issues in the fields of marine environmental variability and climate effects, Zhejiang Ocean University and the South China Sea Institute of Oceanology, the Tidal and Sea Level Professional Committee of the Chinese Society of Oceanography, held the first International Symposium at October 2018. Then the related special issue of Journal of Oceanography and Limnology is collected, to draw attention to some latest researches of marine environmental variability and climate effects in the Asia-Pacific and the Indian Ocean.

The coastal China seas are highly productive areas, with many estuaries, bays, coral reefs and fisheries, and they are the hot spots of global marine biodiversity. Tan et al. (2020) make an assessment on the future changes in marine environment, i.e., sea surface temperature (SST), salinity, dissolved oxygen (DO), sea water $\mathrm{pH}$, and marine net primary productivity (NPP), in the coastal China seas over the $21^{\text {st }}$ century, based on the outputs of Earth System Models. The results show that the East China Seas (ECS, including the Bohai Sea, Yellow Sea, and East China Sea) will be simultaneously exposed to enhanced warming, deoxygenation, acidification, and decreasing NPP as a consequence of increasing greenhouse gas emissions. By the end of this century (2090-2099), SST will increase $3.24 \pm 1.23^{\circ} \mathrm{C}$, the DO, $\mathrm{pH}$, NPP will decline of $-10.90 \pm 3.92 \mu \mathrm{mol} / \mathrm{L}$ (decrease of $6.3 \%$ ), $0.36 \pm 0.02$, $-17.7 \pm 6.2 \mathrm{mg} /\left(\mathrm{m}^{2} \cdot \mathrm{d}\right) \quad$ (decrease of $12.9 \%$ ), respectively, relative to the current levels (19802005). These changes will induce considerable biological and ecological responses, and making the ECS among the most vulnerable ocean areas to future climate change.

As one of the most complicated components of the Earth's climate system, the Asian summer monsoon (ASM), which firstly establishes over the southeastern Bay of Bengal (BOB), affects the social activities and economy in Asia. The effects of spring warm pool in the $\mathrm{BOB}$ on the monsoon onset vortex (MOV) are well discussed, but the feedback of BOB MOV on the evolution of local SST with the barrier layer is still lack of research. In this special issue, Xu et al. (2020) used 2003 spring's NCEP GODAS reanalysis data to revisit how the BOB MOV influences the heat budget in the upper ocean with a barrier layer, and to explore interaction between MOV and BOB SST by consideration of the solar radiation flux penetrating the mixed layer and the existence of barrier layer.

ENSO is another prominent climate signal; it induces extreme weather and influences the global climate system profoundly. It is necessary and important to improve the prediction skills of El Niño events. While the prediction skills of spring predictability barrier (SPB) related El Niño events

\footnotetext{
* Corresponding author: zjuncheng@zjou.edu.cn
} 
depending on the sensitive area's selection, Zhou et al. (2020) defined subsurface of the eastern tropical Indian as the sensitive area. Using the targeted observations to the sensitive area, the SPB-related prediction skills are improved by $20.3 \%$ in general and $25.2 \%$ while added the tropical Indian ocean.

As an air-sea interaction processes, Tropical Cyclones (TCs) intensity change is affected by the ocean. Currently, compared to TC's track forecast, the forecast of TC's intensity accuracy, especially in the western boundary current area. Yu et al. (2020) statistically analyze intensity variety before and after the TCs in the East China Sea (ECS) passing over the Kuroshio. During 1949 to 2016, there are totally 81 TCs passing over the Kuroshio, can be divided as three types, intensified TCs (31), maintained TCs (28) and weakened TCs (22). TCs with the center translational speed among $1-8 \mathrm{~m} / \mathrm{s}$ and the distance from the Kuroshio main axis is $10-75 \mathrm{~km}$ are intensified more significantly. Hu et al. (2020) conduct Typhoon Talim in 2017 as a case study, to investigate the interaction between surface currents and typhoon induced wave distributions. Typhoon Talim's track consistent with the Kuroshio track, the wind-induced current around typhoon eye near Kuroshio can reach to $1.5 \mathrm{~m} / \mathrm{s}$. The root mean square error of significant wave height (SWH) was $0.34 \mathrm{~m}$, the $\mathrm{SWH}$ is positively correlated with high speed wind-induced current. The interaction between surface current and the wind-sea portion of the wave system can influence the distribution of typhoon waves.

The ability of mesoscale eddies to carry drifters has been studied in the northwest Pacific $\left(99^{\circ} \mathrm{E}-180^{\circ} \mathrm{E}\right.$, $0^{\circ}-66^{\circ} \mathrm{N}$ ) during $1993-2015$ by Wang et al. (2020). In most cases, drifters trapped by eddies with daily rotation angle $\leq 90^{\circ}$ around the eddy center, the drifters near eddy centers can be trapped for a longer time, and they tend to travel for a longer time in normalized eddy radii between $0.41-0.76$. In the tropical ocean and Subtropical Countercurrent, cyclones and anticyclones have similar carrying capability, while cyclonic (anticyclonic) eddies have stronger carrying capability in Southern (Northern) Kuroshio Extension. The carrying days are longer when the Rossby number is smaller. Dai et al. (2020) analyzed an observed anticyclonic eddy's spatiotemporal variation of 3D structure and heat, salt and dissolved oxygen transport. This eddy survived for 269 days and the mean radius is $91.5 \mathrm{~km}$, it can reach to $1000 \mathrm{~m}$. The temperature and salinity anomaly cores located at 500-600 m, while the dissolved oxygen anomaly cores located at $250 \mathrm{~m}$. The maximum heat, salt and oxygen transport were $9.37 \times 10^{11} \mathrm{~W}, \quad 3.08 \times 10^{3} \mathrm{~kg} / \mathrm{s}$, and $2.70 \times 10^{2} \mathrm{~kg} / \mathrm{s}$, respectively.

Fan et al. (2020) evaluated the China Ocean Reanalysis (CORA) in the South China Sea. Compared with in situ observational data, such as Argo profiling floats, drifters and cruise data, temporal and spatial distribution characteristics of temperature, salinity, current and mixed layer depths using CORA data are studied. In 50-400 $\mathrm{m}$ in the SCS, the CORA temperature is colder in winter and warmer in summer and autumn, while for the CORA salinity in 0-150 m, it is higher in most time of the year. The mixed layer depths are shallower in both temporal and spatial distribution, for seasonal variations, they are shallower (deeper) in winter (summer). Near the coast, the CORA errors are greater, there exists a weaker SCS western boundary current.

As research in the marine environmental variability and climate effects of the Asia-Pacific and the Indian Ocean, more distinct features of the climate changes, such as summer monsoon, ENSO and TCs, also eddies are revealed. Though some of our data and results are preliminary, we hope publications of this special issue will help to intrigue more interests and researches on climate changes in the Asia-Pacific and the Indian ocean.

As the guest editors of this special issue, we want to thank the publisher, the editors of the Journal of Oceanology and Limnology, Dr. Roger Z. YU, Dr. CHEN Yang, Ms. CHEN Xiaoyu and other colleagues. They have been helping the editorial process and also offering technical support. We also want to thank all the reviewers for their valuable comments that improved the quality of the papers. Many thanks to Zhejiang Ocean University and the South China Sea Institute of Oceanology, the Tidal and Sea Level Professional Committee of the Chinese Society of Oceanography for their support of this special issue.

\section{References}

Dai J, Wang H Z, Zhang W M, An Y Z, Zhang R. 2020. Observed spatiotemporal variation of three-dimensional structure and heat/salt transport of anticyclonic mesoscale eddy in Northwest Pacific. Journal of Oceanology and Limnology, 38(6): 1 654-1 675, https://doi.org/10.1007/ s00343-019-9148-z.

Fan M T, Wang H Z, Zhang W M, Han G J, Wang P Q. 2020. Evaluation of the China Ocean Reanalysis (CORA) in the South China Sea. Journal of Oceanology and Limnology, 38(6): 1 640-1 653, https://doi.org/10.1007/s00343-019- 
9146-1.

Hu Y Y, Shao W Z, Shi J, Sun J, Ji Q Y, Cai L N. 2020. Analysis of the typhoon wave distribution simulated in WAVEWATCH- III model in the context of Kuroshio and wind-induced current. Journal of Oceanology and Limnology, 38(6): 1 692-1 710, https://doi.org/10.1007/ s00343-019-9133-6.

Tan H J, Cai R S, Huo Y L, Guo H X. 2020. Projections of changes in marine environment in coastal China seas over the $21^{\text {st }}$ century based on CMIP5 models. Journal of Oceanology and Limnology, 38(6): 1 676-1 691, https:// doi.org/10.1007/s00343-019-9134-5.

Wang H Z, Liu D, Zhang W M, Li J X, Wang B. 2020. Characterizing the capability of mesoscale eddies to carry drifters in the northwest Pacific. Journal of Oceanology and Limnology, 38(6): 1 711-1 728, https://doi.org/10. 1007/s00343-019-9149-y.

Xu K, Liu B Q, Liu Y, Wang W Q, He Z Q. 2020. Effects of monsoon onset vortex on heat budget in the mixed layer of the Bay of Bengal. Journal of Oceanology and Limnology, 38(6): 1 616-1 631, https://doi.org/10.1007/ s00343-019-9061-5.

Yu Y, Dong C M, Shan H X, Zou B. 2020. Statistical analysis of intensity variations in tropical cyclones in the East China Sea passing over the Kuroshio. Journal of Oceanology and Limnology, 38(6): 1 632-1 639, https:// doi.org/10.1007/s00343-019-9069-x.

Zhou Q, Duan W S, Hu J Y. 2020. Exploring sensitive area in the tropical Indian Ocean for El Niño prediction: implication for targeted observation. 2019. Journal of Oceanology and Limnology, 38(6): 1 602-1 615, https:// doi.org/10.1007/s00343-019-9062-4. 